\title{
Périodicités et Prévisions
}

\section{Periodicity and forecasts}

\author{
PAR A. COU'TAGNE \\ INGENIEUR-CONSEIL \\ Prísidente de la Sectron d'Týrologe

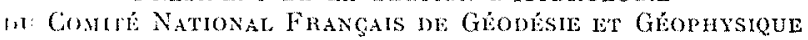

English synopsis, p. 462.

Il fant dire en gros : cela se fait par figure et monvement, car cela est vrai. Mais de dire quel el composer la machine, est ridicule. Car cela est inutile, et incertain, et pénible.

(Pascal.)

Existe-t-il des périodicités, notamment en hydrologie, sur lesquelles on puisse fonder des prévisions présentant une réclle utilité?

Cette question, si controversée, n'est pas pris d'être résolue. 'Tant qu'il y aura des hommes, il y aura des chercheurs de périodes météorologiques et autres, que ne rebuteront ni les difficultés, ni les échecs.

\section{I}

Il y a quelques années, la Revue de Météorologie a ouvert une enquête sur les «périodicités ». Dans les réponses qu'elle a publićes, nous avons glané les quelques appréciations of conclusions suivantes :

\section{d'Anders ANGSTROM :}

La détermination des variations périodiques, de leur amplitude et de leur phase et la probabilité de leur existence réelle constitue un autre problème météorologique assez important. La plupart des phénomènes météorologiques, comme la pluie, la température, le niveau de l'eau, etc., sont sujets à des variations considérables dont la périodicité annuelle el joumalière est en général tout à fait évidente ct déterminée avec exactitude. Mais existe-t-il d'autres périodes et, si c'est le cas, avec quel degré d'exactitude peuventelles être déterminées?

La détermination des périodes était un délassement populaire, chez certains savants, manquant quelque peu d'esprit critique, avant le développement des méthodes statistiques, et surtout avant lapplication de ces methodes à la météorologie et à la géophysique, et il n'existait guère de problemes où ils ne voyaient pas une ou plusieurs périodes. Les recherches scientifiques dans ce domaine, dont le développement est dù peut-être tout autant aux travaux de géophysiciens ayant une solide culture thérique qu'aux progrès de la science statistique pure, ont ordonné et systématisé ce champ de travail, et constitué le terrain objectif qui était nécessaire.

C'est ainsi que l'éminent physicien et mathématicien A. Schustrer a établi, pour l'élude des variations périodiques, une méthode qui introduit une comparaison entre, d'une part, l'amplitude de la période dont on suppose l'existence, ct, d'autre part, une amplitude théorique, l'expectative, qui est ensuite déterminée à l'aide du calcul des probabilités.

Parviendrons-nous un jour à une connaissance de tous les facteur's météorologiques el de leurs relations mutuelles suffisante pour nous permettre -- par exemple, d'apres notre connaissance de $x, y, u \ldots$ - d'obtenir une valeur exacte d'y et de ses variations futures? Nous pouvons espérer une réduction de notre marge d'errents, par l'introduction de nouveaux facteurs dans notre système d'observations météorologiques; cependant le nombre des facteurs qui échappent it nos mesures demeurera nécessairement si grand que nos prévisions se réaliseront toujours avec un certain degré de probabilite, non avec certitude.

\section{de BRAZIER :}

L'origine de la croyance à la périodicité des anomalies météorologiques est extrêmement an- 
cienne et il est vaisemblable que cette croyance a été ancrée dans l'esprit humain par le retour périodique des saisons... Une induction loute naturelle devait lamener lòt ou tard ì expliquer les anomalies des saisons par des considérations du même ordre, et il est probable que le pas fut vile franchi. C'est là sans doute que se trouve le point de départ de toules les lentatives qui ont été faites en vue de démontrer la périodicité des fluctuations climatiques, et de relier ces dernieres aux mouvements des astres.

L'hypothèse qui lui sert de base est si séduisante qu'elle a résisté à tous les échees. On peut même dire qu'elle est passée à l'état de croyance et qu'à l'heure actuelle rien n'a pu entamer la foi populaire dans l'influence prépondérante de certain satellite de la Terre sur les anomalies du temps.

Il n'y a qu'un seul astre et deux cycles astronomiques dont laction sur la météorologie terrestre est indéniable.

En toute impartialité et en toute modestie nous sommes obligés de reconnâtre que la série d'observations dont nous disposons ne nous permet pas de trancher par oui ou par non la question d'une correspondance éventuelle entre les pluies et l'activité solaire dans la région parisienne.

Celui qui croit qu'une relation existe entre deux phénomènes ne peut pas démontrer que le partisan de l'opinion contraire est dans l'erreur, et reciproquement. L'existence de cette relation est donc une simple question de croyance. Mais croyance n'est pas science, et pour prévoir juste et sans se tromper, une croyance ne vaudra jamais une loi bien démontrée.

Les voies que peut suivre le chercheur de périodes sont jalonnées dembuches.

\section{de DEDEBAND:}

D.uns les recherches sur les variations séculaires ou périodiques du temps et des climats, il est à peu près indispensable de choisir des éléments méléorolngiques ayant un caractère synoptique, e'est-i-dire dont les valeurs soient représentatives de l'état d'une grosse « particule » d'air environnant le point d'observation. Certes, on peut concevoir à la rigueur qu'on puisse mener ces recherches à partir d'un élément météorologique influencé par les condilions locales, mais à la condition que le point d'observation n'ail jamais été déplacé, et que les environs n'en aient jamais été modifiés de quelque maniere que ce soit.

Des éléments comme la pluie el la tempéralure sont par conséquent impropres aux recherches sur les variations systématiques des climats.
La pression barométrique parait au contraire l'élément le plus convenable.

La pluviosité se rattache à la variabilité de la pression. Celte corrélation est forte sauf pendant les mois d'été et elle s'améliore d'ailleurs si on synoptise la pluie, c'est-à-dire si l'on fait la moyenne des précipitations recues par une région assez étendue.

Malheureusement l'appel aux éléments directs limite les investigations à la période assez courte depuis laquelle on fait des observations météorologiques suivies. On concoit que dans ces conditions l'ingéniosité des chercheurs se soit donné libre cours pour reconstituer les climats passés à l'aide de témoins. L'interprétation des indications données par ces témoins est évidemment très délicate, et doit faire, dans chaque cas particulier, l'objet d'une analyse critique. Le niveau des lacs et mers fermées traduit une résultante complexe d'effets de pluviosité, d'évaporation, de ruissellement, d'infiltration intéressant une région très étendue qui peut comprendre plusieurs zones climatiques dans lesquelles les variations soutenues ne sont pas nécessairement de même sens.

Les « souvenirs historiques » d'hivers très froids, d'étés très chauds, d'inondations, lorsqu'ils sont dûment contrôlés par une analyse synoptique, ont l'inconvénient de n'indiquer que des paroxysmes, et de laisser échapper les variations lentes et soutenues, tout aussi, sinon plus intéressantes.

L'analyse harmonique n'est bien entendu qu'une pure opération mathématique qui conduit senlement $\dot{a}$ une représentation absolument artificielle d'une courbe quelconque, au moyen d'ondes sinusoïdales dont les périodes, multiples les unes des autres, dérivent de l'intervalle d'analyse choisi. Cette représentation n'est pas le moins du monde extrapolable. Pour qu'elle soit extrapolable, il faut que les composantes aient une réalité physique, comme c'est le cas pour les ondes de marée, par exemple.

Pour juger de la réalité des ondes trouvées, il existe des critéres mathématiques, basés sur le calcul des probabilités, mais les renseignements donnés par le caleul des probabilités ne commencent à donner des quasi-certitudes que s'íls reposent sur de grands nombres.

On peut faire appel aussi à des critères expérimentaux.

Bien que les chercheurs de périodes aient déployé des efforts nombreux et considérables, il ne semble pas que dans les quelques centaines de périodes météorologiques découvertes jusqu'à ce jour il $y$ en ait de réelles, en dehors de la période diurne et de la période annuelle.

C'est cet insuccès persistant qui a sans doute amené les cyclomanes à étendre le mot onde 
et à rechercher des oscillations moins élémentaires que des sinusoüdes.

Les insuccès répétés des chercheurs de périodes montrent, sans contredil possible, que la partie systémalique des variations du temps el des climats est faible par rapport à la partic aléatoire. Ceci diminue singulièrement l'intérêt pratique, pour la prévision du temps à longue échéance, en particulier, que pent avoir la découverte d'une période même réelle, et nous n'en sommes pas encore là, si minutieusement dissimulée au sein des variations accidentelles qu'il faut pour la mettre en évidence des manipulalions malhématiques compliquées des matériaux bruts d'observation.

On peut craindre que les effets d'une périodicité si alténuée soient sans importance pratique au point de vue social ou économique.

\section{de M.K. STUMPFF :}

Les variations annuelles et diurnes des éléments météorologiques sont comnues; ce sont les seules pour lesquelles il existe une explication théorique sure : l'oscillation annuelle et diurne de l'insolation due à des mouvements cosmiques.

Dans les régions à climat simple et constant (et notamment les régions tropicales maritimes) ces variations contribuent d'une façon prépondérante à la formation du temps. Dans les zones arctiques et tempérées, par contre, les écarts à cette marche régulière, généralement désignés comme apériodiques, ont une prépondérance et un ordre de grandeur tels que le mouvement périodique n'apparaît plus que dans les moyennes relatives à de très grands intervalles de temps. Çe sont généralement ces écarts apériodiques qui caractérisent à nos latitudes le temps et ses variations irrégulières qui surviennent souvent avec une rapidité surprenante.

En ce qui concerne les précipitations, toutes les méthodes qui sont basées sur l'analyse des phénomènes discontinus sont inutilisables. La recherche de périodicités dans des mesures pluviométriques ne peut être entreprise, tout au plus, que lorsqu'on dispose de sommes ou moyennes relatives à des espaces de temps d'une assez longue durée, elles n'acquièrent done une certaine importance que lorsqu'il s'agit de la recherche d'oscillations de grande longueur d'onde.

\section{de WALKER :}

Lorsque l'analyse harmonique a mis en évidence une amplitude finie correspondant à une certaine période, le problème fondamental est d'étudier si cette période est réelle et due à des causes physiques, ou bien fictive ou accidentelle. Si la probabilité d'une telle amplitude, dans une série de nombres pris au hasard, est suffisanment grande, la périodicité n'est pas réelle.

On peut regretter que la plupart des chercheurs de périodicités semblent montrer une certaine répulsion pour l'analyse de Fourner.

L'épithète «psezdo-périodique » semble etre principalement employée par ceux qui ne peuvent trouver une période réelle dans la pratique, et espèrent, par un titre plus vague, ponvoir prétendre à un progrès dans cette citude.

L'auteur ne connait pas de période dont l'anplitude soit suffisamment grande pour justifier son emploi en prévision à longue échéance. Mais il accepte pleinement la suggestion que des piriodes réelles de petite amplitude puissent ètre de grande importance scientifique, en nous donnant des renseignements à la fois sur les variations internes de l'atmosphère et sur les influenecs extrêmes auxquelles elle est sujetle.

\section{II}

Ces quelques extraits bibliographiques donnent lieu, de notre part, aux déductions ef commentaires suivants :

1. S'il existe de multiples périodicités cautres que le cycle journalier et le cycle annuel et peutêtre le cycle, plus ou moins fluctuant, des taches solaires), il apparaît difficile de les déceler, compte tenu de la très faible durée des observations que nous possédons. Une statistique portant sur 50-100 années est absolument insuffisante, en l'occurrence, s'il s'agit de périodes d'une certaine durée.

Même si de telles périodicités étaient trouvées, et reconnues réelles et non fictives, elles seraient de peu d'utilité pour la prévision de tel ou tel facteur hydrométéorologique ou autre, étant donné que la partie systématique des variations en jeu est faible par rapport a la parlic alćatoire.

2. Toutes les méthodes de décomposition analytique d'une sinusoïde irrégulière expérimentale en plusieurs composantes, dont le nombre est d'ailleurs indéterminé et varie au gré de la fantaisie de chaque crelomane, sont plus ou moins subjectives. On procède en définitive par tâtonnements successifs ou par intuition personnelle. Si l'on soumettait une série statistique à des calculateurs différents, utilisant ou non des méthodes d'investigation différentes, et opérant sans idées préconçues, il est certain que les solutions proposées seraient également différentes les unes des autres.

3. Bien des arguments, fondés par exemplo sur des considérations simples de probabilités, 
bien des constatations expérimentales, bien des considérations inspirées par le bon sens, peuvent être invoqués contre l'existence de telles multiples périodicités.

Ainsi, dans la suite des années, il y a autant de chances de voir succéder, à une amnéc humide, par exemple, une année humide ru'une année sèche (dans certaines régions toüt au moins). Aussi bien, si l'on faisait le dénombrement de toutes les périodicités proposées, on en trouverait plusieurs centaines... Conclure à de très nombreuses périodicités ou dire qu'il n'y en a pas, c'est pratiquement la même chose. Une variation résultant d'une multitude de causes, dont aucune n'est apparemment prépondérante, rentre dans la catégorie des choses que nous appelons «aléatoires».

4. Un argument de fait réside dans les nombreux échees éprouvés par les chercheurs de périodes dans leurs prévisions.

\section{Nous en citerons quelques-uns :}

- Le 11 mars 1921, on pouvait lire dans le Petit Journal, sous la signature de l'abbé Morevx : «Encore quatorze ammées de sécheresse, voilà ce que permet d'affirmer l'étude du soleil. » La période de sécheresse ainsi prévue a été, en réalité, la plus humide que l'on ait enregistrée depuis cent ans! En Europe, tout au moins, car dans certaine région montagneuse des Etats-Unis elle a été catastrophiquement déficitaire en précipitations.

- Hutchins avait élabli l'existence d'une période de neuf ans dans la succession des pluies en Afrique du Sud, celles-ci croissant pendant quatre ou cinq ans puis décroissant pendant le même laps de temps. Appliquée à une période ultéricure, cette loi périodique s'est trouvée complètement en défaut.

- On peut citer encore les travaux de M. WALLEN sur les variations du niveau des grands lacs (Suède), ainsi que les prévisions à longue échéance publiées par ce savant et basées sur la méthode de Scmremer. D'après l'auteur, ces prévisions devaient donner les meilleurs résultats pour les cours d'eau au régime lacustre. On remarquera cependant que, même pour les lacs, elles ont donné lieu à une discordance grave entre les niveaux calculé et réel pour l'année 1921 caractérisće par une sécheresse exceptionnelle.

- A citer également la prévision établie par Stremr, météorologiste américain, pour les niveaux du Dniepr. Au lieu de labaissement des niveaux prédit pour 1931, on observa sur ce fleuve une crue qui a dépassé tous les maxima connus.
5. Aussi bien la notion de «période » proprellkent dite a été peu à peu abandonnée. On a d'abord parlé de psendo-période, puis d'onde plus ou moins sinusö̈dale, à amplitude, phase, période variables. De là à la notion pure et simple de flactactions plus ou moins aléatoires, donc apériodiques, il n'y a qu'un pas à franchir.

Certes, de telles fluctuations sont indiscutables. Mais - même si elles se reproduisent à intervalles plus ou moins fixes, on ne peut baser sur leur existence aucune prévision d'un récl intérêt.

6. S’il y a des causes premièes, périodiques, d'ordre cosmique, agissant plus ou moins indirectement sur la température, la pluie, le débit des cours d'eau, etc., elles doivent agir ainsi, non pas isolément sur tel ou tel point de l'univers, mais, sinon sur la terre entière, du moins sur une portion assez étendue de cette terre. D'où la nécessité - surtout si l'on veut discriminer ces causes premières des facteurs locaux - de soumettre à l'analyse non pas les données statistiques d'une seule station, d'un seul cours d'eau, mais les données statistiques moyennes de nombreuses stations.

Les périodes de sécheresse, par exemple, même pour des régions assez voisines, ne coincident jamais absolument. Ainsi, l'année 1921, particulièrement sèche en France, a été, en certains points de l'Europe, humide et parfois même la plus humide d'une série de cinquante années successives.

De même, la période spécialement pluvieuse que nous venons d'avoir, qui semble avoir pris fin en 1941, a été très déficitaire en pluie dans la Sierra Nevada.

«Pour déterminer l'influence des taches solaires sur la température, a écrit M. Jean Bosr.rn, il ne suffit pas de noter les températures en un lieu de la terre, à Marseille, par exemple, et de constater que durant plusieurs eycles de la période undécennale des taches les années chaudes ont cöncidé avec celles oì le soleil avait le plus ou le moins de taches. La terre est grande : il n'y a pas que la température cu'il fait à Marseille qui entre en jeu : il faut aussi tenir compte de celle qu'il fait à Londres, à Buenos-Aires, à Melbourne, etc., et faire porter les statistiques sur le globe entier, eu égard aux surfaces des pays envisagés, sans même en excepter les déserts et les océans, et ceci complique singulièrement l'affaire. Si longue, si fastidieuse et si difficile à bien exéculer que soit cette statistique, elle a pourtant été entreprise. On a reconnu que, dans l'ensemble, les années chaudes sont celles où les taches solaires passent par un minimum. La différence est très faible; la température moyenne du globe est tout an plus de quelques 
dixiemes de degré plus forte durant ces annéeslà : mais le sens de l'écart semble établi, et c'est beaucoup. Ce sens dément cependant l'opinion générale : on croit d'ordinaire que les taches amènent la chaleur, or c'est le contraire qui est vai. Le résultat trouvé parait dụ resle conforme au bon sens. Les taches sont en effet des régions moins chaudes de la surface solaire. Il est donc naturel que le soleil nous envoie relativement moins de chaleur quand il a beatucoup de taches que quand il en a peu. »

Telle est, du moins, l'opinion émise par M. Boster. Mais les avis sont, paraît-il, partagés quant à dire que le maximum de température corresponde au minimum ou au maximum des taches solaires, et nul n'est certain qu'un maximum thermicpue à la surface de la troposphère se traduise par un maximum thermique à la surface du sol.

7. En ce qui concerne plus spécialement les facteurs hydrologiques, si une périodicité cosmique première agit sur le régime des eaux à la surface de la terre, elle agit d'abord, nécessairement, sur les précipitations, puis clle se répercute, plus ou moins amortie ou amplifiée, déformée - parfois même ses effets se trouveront annulés - sur les débits moyens, puis éventucllement sur chaque débit individualisé, par exemple le débit de crue, c'est-à-dire le plus fort débit instantané de l'année: un tel débit peut d'ailleurs être faible au cours d'une année tris pluvieuse.

Il y a des bassins pou: lesquels on ne constate aucune corrélation sensible entre lindice pluviométrique et l'indice d'écoulement annuels, étant donné la prépondérance, sur l'écoulement, d'antres facteurs que la pluie (temperature, rétention, etc.). Ainsi en est-il, notamment, des cours d'eau glaciaires et des bassins à faibles précipitations (le Dniepr, par excmple).

Si l'on veut étudier de telles périodicités, il faut donc étudier l'élément témoin le plus directement influencé par les causes premières, done les précipitations. Si l'on considère les débits, il faut qu'il y ait corrélation entre les dóbits el les précipitations et considérer de préférence de grands bassins totalisant les precipitations moyennes d'une région assez étendue. Le choix des debits de crue est plus discutable : nos connaissances et nos prévisions ne peuvent porter que sur des ensembles et non sur des individualités. Y a-t-il quelque chose de plus individualisé ct apparemment plus aléatoire que le plus fort débit de l'annće?

Il nous paraît done chimérique d'espérer prévoir les périodes d'inondation de tel ou tel cours d'anu, de mène que les grands hivers dont la stalistique est d'ailleurs difficile à établir, car ello repose en grande parlie sur des " souvenirs historiques $\gg$. Il suffit de rappeler, à ce sujet, les travaux de labbé Gabries (cycle Iuni-solaire de 186 ans) et la eritique qu'en a faite M. Braziñ. Antérieurement, il y a plus d'un siècle, Lasalus, traitant le mème sujel, avait conclu à une périodicité de cent ans.

Le comte de Gasparrs, après avoir rendu compte du mémoire de cet auteur, en tirait les conclusions suivantes : "Nous comptons $116 \mathrm{hi}$ vers rigoureux dans un espace de 543 ans : c'est un grand hiver tous les quatre ou cinc ans. De plus, nous voyons que souvent trois hivers rigoureux se succedent sans interruption : 14321433-1434; 1522-1523-1524, etc. Nous voyons aussi que les sciries des hivers doux et rigoureux sont irrégulières et plus ou moins prolongées, et qu'ainsi, dars nos climats, on doit toujours ctre en mesure de les braver par les précautions que conseille une complète connaissance des végélaux. Cette observation s'adresse surtout à ceux qui, placés aux limites des régions agricoles, doivent craindre plus que les autres les saisons excessives. C'est a eux que nous recommandons de ne croire da aucun pronostic qui leur annoncerait un hiver doux, mais d'agir toujours comme si l'hiver devait être rigoureux. »

Sages paroles, pleines de bon sens, qu'il est bon de méditer. La seule prévision possible, en la matiere, est une probabilité : un hiver rigonreux sur cinc en moyenne.

Nolons incidemment que nous venons de traverser une période de cinquante ans où cette proportion a été beaucoup plus faible. Effectivement, depuis le debut du siècle, les hivers ont cté particulierement doux, et les écarts thermiques, d'une année à l'autre, atténués.

C'est ce que montre le tableau suivant des températures moyennes et des précipitations du mois de janvier (Lyon-Fourvière) établi par décade :

\begin{tabular}{|c|c|c|}
\hline & Temperature & Précipitation \\
\cline { 2 - 3 } & \\
\hline $1890-1899 \ldots$ & $0^{\circ} 966$ & $23,91 \mathrm{~mm}$ \\
$1900-1909 \ldots$ & $1^{\circ} 241$ & $27,06 \mathrm{~mm}$ \\
$1910-1919 \ldots$ & $1^{\circ} 617$ & $20,14 \mathrm{~mm}$ \\
$1920-1929 \ldots$ & $2^{\circ} 470$ & $30,42 \mathrm{~mm}$ \\
$1930-1939 \ldots$ & $.3^{\circ} 323$ & $41,01 \mathrm{~mm}$ \\
\hline
\end{tabular}

II y a là une «fuctuation elimatique » très nette : hivers de plas en plus chands, et par suite de plus en plus humides (1). On peut en

(1) Un hiver froid est généralement sec et inversement - mais pas toujours. Ainsi, les cind annees $1940-44$ contredisent eette loi et sa régularité (température et pluies du mois de janvier a tyon: ' $\mathrm{T}:-0,20$ et $\mathrm{H}: 52 \mathrm{~mm}$. 
ciler une autre, relative à la mème période : le recal persistant des glaciers dans les Alpes.

\section{III}

Etant donné la subjectivité de toute analyse harmonique, il est indispensable de soumcitre à un critérium objectif toutes les lois périodiques mises en avant, soit pour en vérifier l'existence probable, soit pour en apprécier le regré d'exactitude ou d'approximation, tout all moins pour la période qui a servi a son établissement. Il restera, ensuite, à en contròler l'exactilude dans les périodes suivantes.

Un critérium simple, élémentaire, résulte des considérations et calculs suivants, valables d'aitleurs pour apprécier, et mème chiffrer, la valeur d'une corrélation quelconque, périodique ou non, entre deux ou plusieurs facteurs geographiques ou autres. Ine loi de périodicité n'est cu'une corrélation entre un facteur géographique et le temps.

Soit $X$ la variable statistique considérée, $X_{0}$ sa moyenne, et $\mathrm{X}^{\prime}=f(t)$ la corrélation périodique à vérifier.

Cette corrélation est d'autant plus probable que les points observés se groupent mieux au voisinage de la courbe $\mathrm{X}^{\prime}=f(t)$ qu'au voisinage de la droite $\mathrm{X}=\mathrm{X}_{0}$.

J'appelle écart moyen de probabilité l'expression :

$$
\mathrm{E}=\frac{\Xi\left|\mathrm{X}-\mathrm{X}_{0}\right|}{n}
$$

et écart de corrélation l'expression :

$$
e=\frac{ \pm\left|X-X^{\prime}\right|}{n}
$$

Con pourrait tout aussi bien considérer les écarts quadratiques moyens, mais les calculs seraient inutilement plus longs).

Plus e sera petit par rapport à E, plus la corrélation sera forte et utile à considérer, en vue notamment de prérisions éventuelles.

L'expression $r=1-\frac{e}{\mathrm{E}}$, que j'appelle degré d'exactitude et plus simplement degré de la corrélation - gui traduit un rendement, permet de chiffrer - de 0 à 1 - la précision d'une corrélation, et notamment d'une corrélation périodique.

Les deux exemples suivants illustrent ces considérations :

1. Le cycle annuel est une réalité, dont l'influence se manifeste plus on moins suivant les facteurs et les régions. D'où la notion d'année moyenne sur laquelle repose d'ailleurs toute la climatologie statistique. Mais cette notion est plus ou moins exacte suivant le facteur et la région, suivant que l'on considère notamment, par exemple, les régions tempérées ou tropicales, la pluie ou la température.

Dans les régions tempérées, c'est, comme on sait, l'élíment température qui est le plus en har-

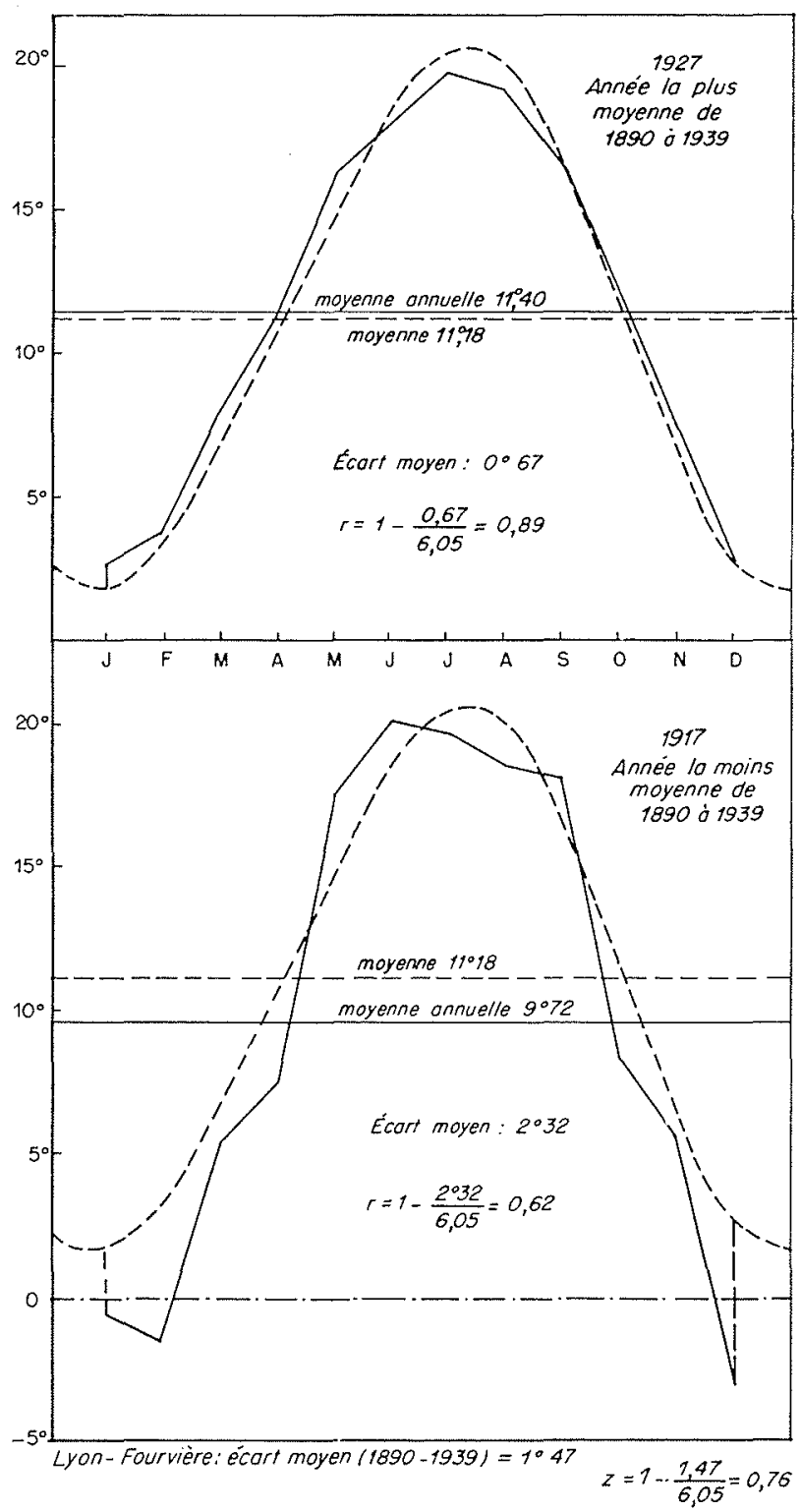

GraphIOUE I

monie avec le cycle solaire annuel. Ainsi pour Lyon, où la température moyenne annuelle (Lyon-Fourvicre, 1890-1939) est de $11^{\circ} 18$, les températures mensuelles observées se situent nettement mieux autour de la sinusoïde moyenne constituce par les températures mensuelles moyennes, qu'autour de la droite moyenne :

$$
\mathrm{T}=11^{\circ} 18 \text {. }
$$

On a effectivement, en employant les notations indiquées ci-dessus : 
$\mathrm{E}=6^{\circ} 05, e=1^{\circ} 47$ et $r=1-\frac{1,47}{6,05}=0,76$.

Nous avons, pour la série d'années considérées, tracé les graphiques correspondant à l'année la plus moyenne (1927) et à l'année la moins moyjenne (1917) caractérisées par les écarts respectifs $e=0^{\circ} 67(r=0,89)$ et $e=2^{\circ} 32(r=0,62)$ (graphique 1 ).

On se trouve bien en présence d'une périodicité réelle.

Si l'on considire le facteur pluie, il n'en est plus de même, et pour la France le degré de corrélation est de 0,10 à 0,20 . Il sera par contre de 0,62 à Addis-Abéba, en Ethiopie.

En ce qui concerne les débits, dont la répartition saisonnière est conditionnée à la fois par la température et par la pluie, la notion d'année moyenne, en France, sera d'autant plus exacte, par conséquent, que le débit sera plus conditionné par la température que par les précipitations. La considération du degré de corrélation confirme ce fait. On trouve, par exemple :

$r=0,82$ pour l'émissaire du glacier d'Aletsch.

$r=0,61$ pour la Romanche (alt. moy. $2.140 \mathrm{~m}$ ).

$r=0,38$ pour le Dorinet (alt. moy. $1.800 \mathrm{~m}$ ).

$r^{\prime}=0,20$ pour la Truyère.

Les prévisions de débils, basées sur leurs probabilités mensuelles, sont d'autant plus aléatoires que le bassin a son alimentation plas exclusivement pluviale. C'est là un fait bien connu de tous les exploitants d'usines hydrauliques, des Alpes et du Massif Central.

$2^{\circ}$ Dans une etude sur la Pluviosité au Portugal. M. A. de Carvalho a procédé à l'analyse har- monique (méthode Fovnien) des données pluviométriques d'une station, celle de Serra da Estrella, la plus pluvieuse du pays $(\mathrm{H}=2.420 \mathrm{~mm}$ ). Les calculs l'ont conduit à trois périodes : $36-18$ et 12 ans. La sćrie d'observalions porte sur 50 années.

Le graphique II, que nous avons déduit de ces calculs, montre que les points observés se groupent mieux, évidemment, autour de la sinusoïde tripartite présumée, mais pas tellement.

On a effectivement :

$$
\begin{aligned}
\mathrm{E} & =629 \mathrm{~mm} \\
e & =492 \mathrm{~mm}
\end{aligned}
$$

d'où : $\quad r=0,22$.

C'est là un degré de corrélation plutôt faible.

En d'autres termes, en prévoyant comme pluviosité d'une année à venir une pluviosité égale à la moyenne statistique 2420 , on risque une erreur moyenne de $26 \%$ (en pour cent de ladite valeur moyenne). Cette erreur est abaissée à $20 \%$ si on utilise la loi périodique proposée.

Les taux respectifs sont même de $28 \%$ et $25 \%(x=0,10)$ pour les vingt-cinq dernières années de la série.

On peut, dans ces concialucas, mettic en doute la réalité des trois périodes décelées par l'analyse. L'auteur en convient d'ailleurs parfaitement « attendu la petitesse de la série, on ne peut pas dire sûrement si c'est le cas d'une simple coïncidence, ou le fait d'un phénomène réel ». Même si les périodes étaient réelles, aucune prévision sensible n'en résulterait, le gain de probabilité réduisant seulement de $26 \%$ à $20 \%$ (et même de $28 \%$ à $25 \%$ ) l'erreur moyenne.

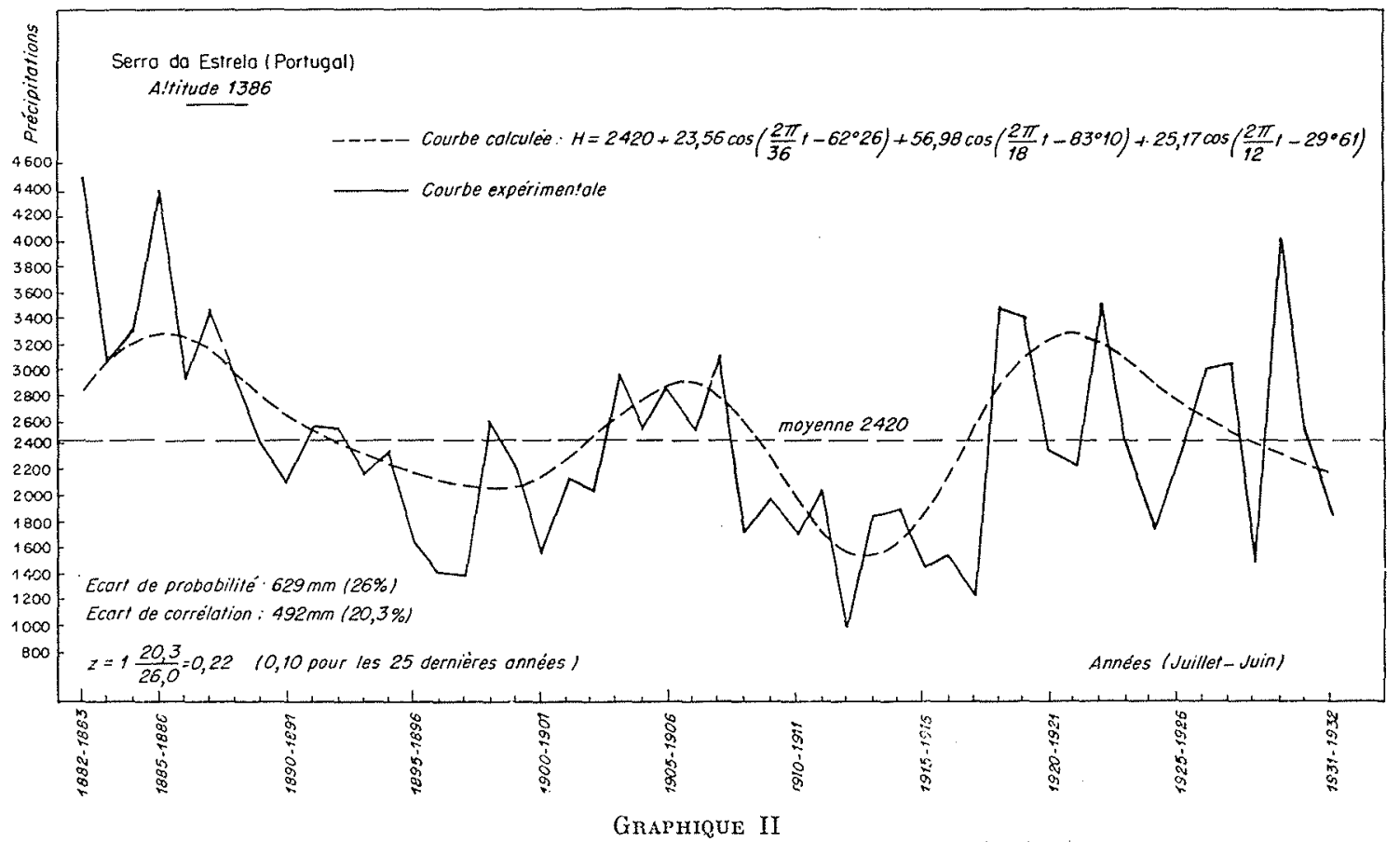


Parmi les méthodes graphiques élémentaires cmployées pour mettre en relief les variations périodiques, les fluctuations, les tendances d'un facteur géographique, on peut citer la méthode d'adoucissement par sommalion dordonnes ou des moyennes chevanchantes, et la méthode dite des écarts cumulés.

La premiere est le prototype de divers procédés appliqués depuis longtemps par les météorologistes, et la méthode Labrouste en constitue la généralisation méthodique et rationnelle.

Nous en donnons diverses applications (graphiques III, IV et V) d'où l'on peut tirer quelques enseignements relatifs à la période dite de Bruchner et à la période undécennale, les deux principales périodes dont l'existence a été suggérée en hydrologie.

1. Les précipitations et la période des taches solaires dans la Sierra Nevada.

Le graphique III juxtapose les deux courbes obtenues en faisant la moyenne des données annuelles, 5 par 5 , puis la moyenne 3 par 3 des chiffres ainsi obtenus.

Les précipitations dont il a été fait état sont les moyennes de 6 stations de la Sierra Nevada Centrale (U.S.A.) dont l'altitude varie de 1.360 à 7.017 pieds.

Après trois oscillations assez régulières, et plus ou moins en harmonie avec celles des taches solaires, il y a ensuite discordance complète : à deux maxima de cette dernière courbe correspondent deux minima, peu marqués d'ailleurs, de la courbe des pluies, en décroissance générale très accentuée depuis 1906 .

Il n'y a pas de corrélation, en définitive, entre les précipitations et les taches solaires dans cette région.

Ce résultat est à rapprocher des conclusions de

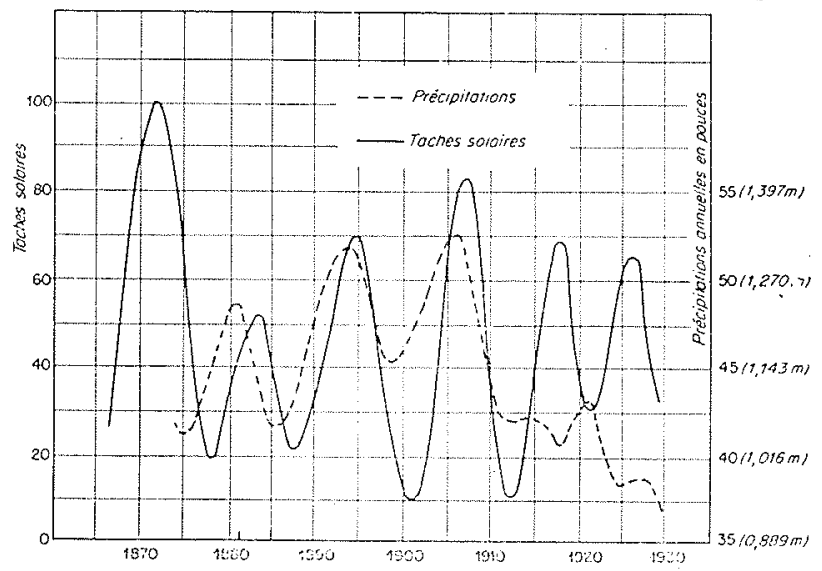

Grapmote III

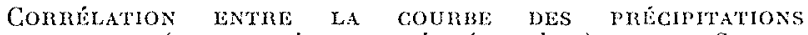
ANNUELLES (moll. quinquennales-ternaires) DE LA SIERTA Nevada centrale $(6$ stations) et IA counie comResponDANTE DES TAGHES SOLAIRES.
GRAPHOUE IV
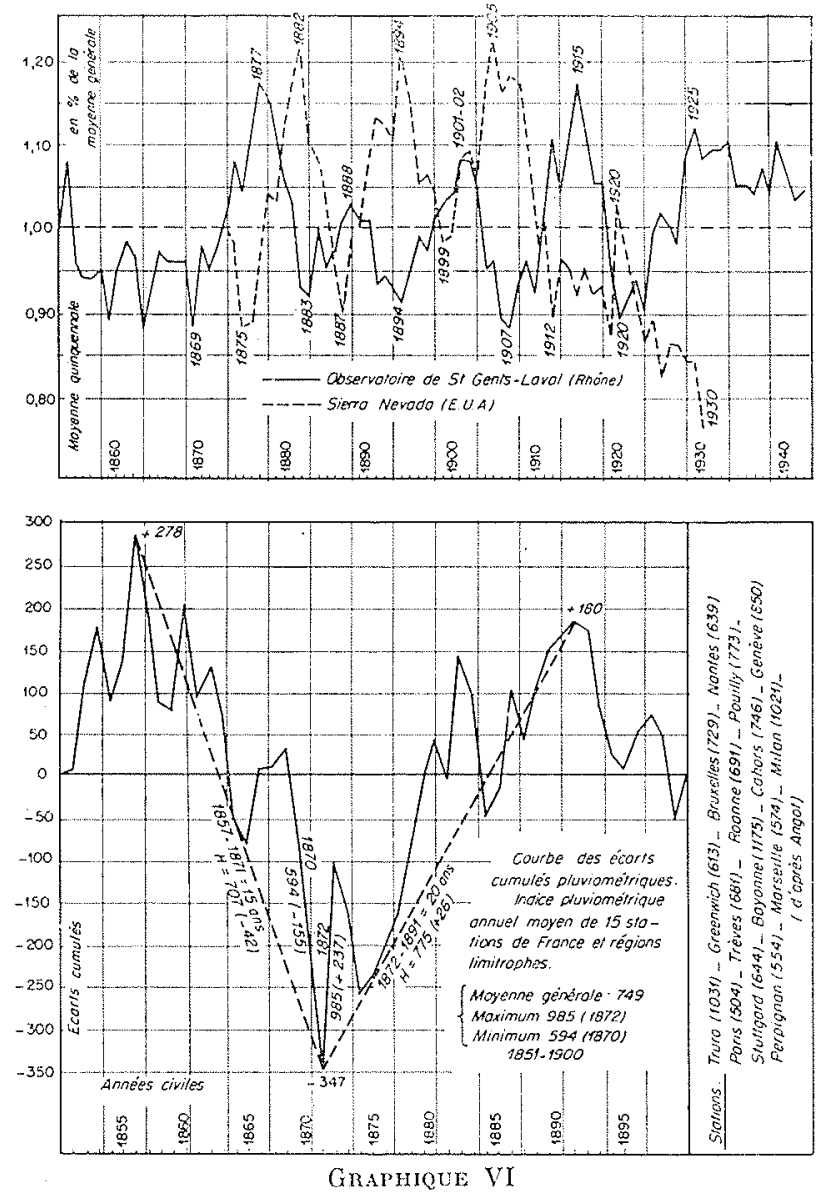

M. Brazier sur le même sujet, en ce qui concerne la région parisienne, dont nous avons fait mention plus haut.

2. Comparaison entre les pluies de la Sierra Nevada et celles de la région lyonnaise.

Le graphique IV montre qu'il y a discordance complète entre les fluctuations périodiques do ces deux régimes.

On note bien une alternance de onze à qua torze ans entre les premiers maxima $(1877-1888$ 1902-1915-1929) et minima (1883-1894-19071920) de la région lyomaise - et également entre certains maxima et minima de la Sierra Nevada, mais de là à conclure à la réalité d'une périodicité épousant celle des taches solaires, il y a loin : les dernières années contituent pour la Sierra Nevada une période particulierement sèche, et pour la région lyonnaise une période particulièrement humide, sans corrélation avec le nombre des taches solaires.

Signalons incidemment que la méthode de corrélation appliquée aux clonnées pluviométriques de Lyon et d'un bassin des Montagnes Rocheuses (Skagit River) pour vingt-cing années, donne un rapport de corrélation assez faible, mais négatif $(\mathrm{R}=-0,38)$. 


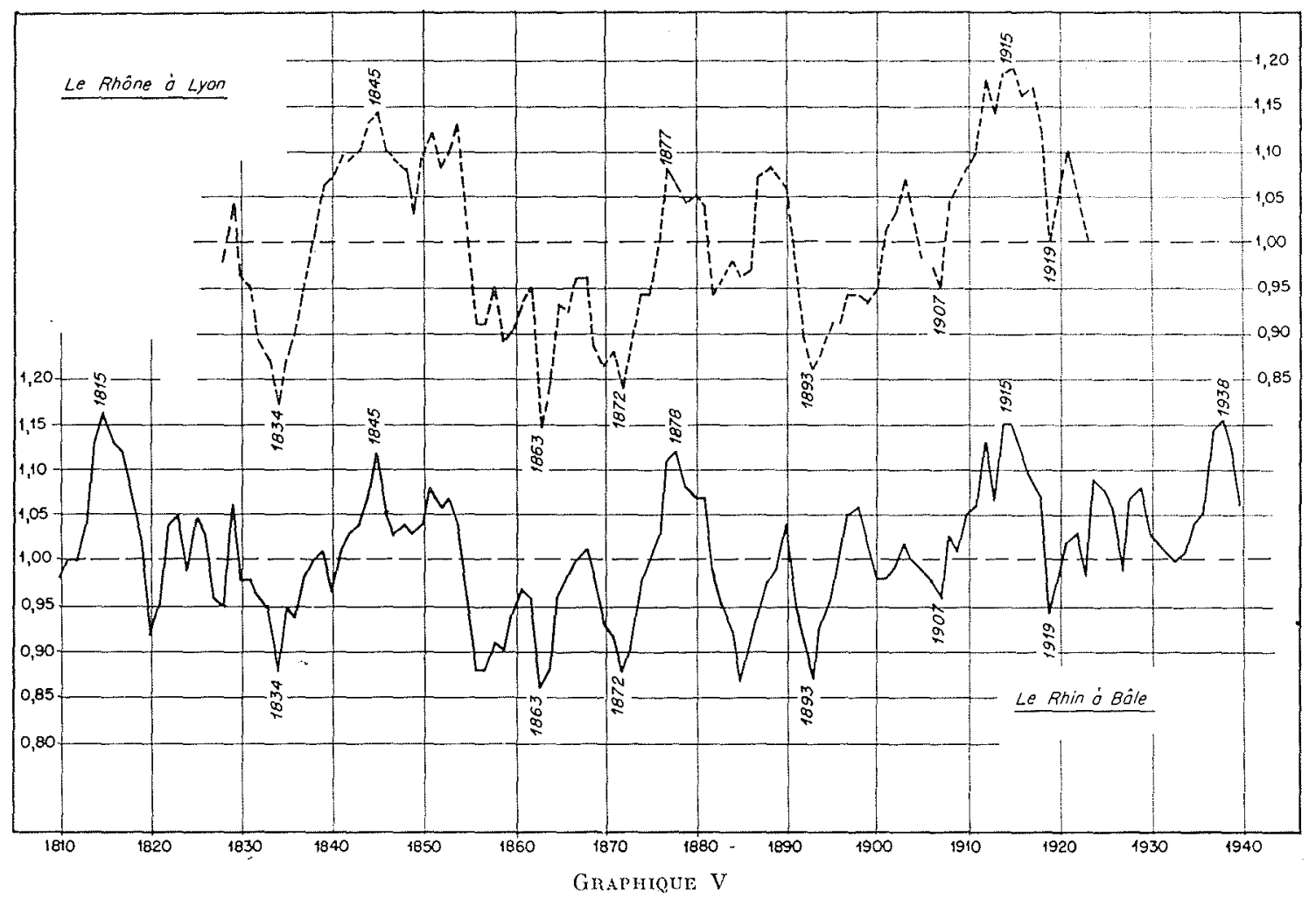

3. Comparaison entre les variations periodiques du débit du Rhóne à Lyon et du débit du Rhin a Bâle (graphique V).

Dans l'ensemble, ces variations apparaissent assez synchrones et mettent on relief - de 1810 à 1940 - 5 périodes pluvieuses centrées (moyenne quinquennale) sur 1815-1845-18781915-1938, alternant avec des périodes seches centrées sur 1834-1863-1893-1919. Il nous parait osé de tirer des intervalles correspondants, soit 30 33-37-23 et 29-30-26 ans un argument en faveur de la période Bruckner.

Un graphique analogue -- celui du Danube à Orsova - fait apparaitre les mèmes fluctuations générales, et notamment les maxima de 1845 . 1878 et 1915 .

Nous concluons seulement de ces observations que les variations périodiques des cćbits du Rhin, du Rhône ct du Danube sont en corrélation, et que l'Europe Centrale constitue un ensemble assez cohérent du point de vue pluviométrique.

Notons d'ailleurs que la méthode de corrélation appliquée aux débits annuels du Rhône el du Rhin (1826-1925=100 ans) donne un rapport de corrélation sensible: $\mathrm{R}=0,83$.

En ce qui concerne la méthode des ćcarts cu. mulés, nous donnons le graphique VI des Précipitations de la France et régions limitrophes pour une période de 50 ans $((1850-1900)$. Il a été fait état de 15 stations. Ce graphique met en évidence une période de 35 ans, comprenant une période sèche de 15 ans (1856-1871), suivie d'une période humide de 20 ans (1871-1891).

Un graphique analogue, mais établi pour la seule station de Lyon, pour les années ultérieures, donne, par contre, une période sèche de 18 ans (1891-1909), suivie d'une période humide de 26 ans.

\section{$l^{\prime}$}

Des travaux récents ont attiré l'attention sur l'existence de certaines périodicités (autres que la période annuelle dont linfluence de ce point de vue ne fait aucun doute) dans les manifestations de la vie animale et végétale, et établi certains rapprochements entre ces périodicités et celles du monde solaire.

La période undécennale des taches solaires et l'accroissement des arbres.

Nous extrayons les considérations qui suivent d'un article de Jean Bosler, paru en 1937 dans le Bulletin annuel de la Commission de Météorologie des Bouches-du-Rhône sous le titre "Taches solaires et Climats des temps passés Météorologie sans observateurs avant l'apparition de l'homme $»$.

«Vers 1901, divers chercheurs et principalement en Amérique M. A. E. Doughas, se sont apereus que dans certains pays les anneaux de certaines espèces d'arhres présentaient, au point de vue de la largeur, des alternances fort risibles : lidée vint alors que la période de ees dernières pourrait coïncider avec celles des taches 
solaires. Les vérificalions entreprises aussitol dans cetle voie ne tarderent pas à ètre couronnées de suecès. C'est ainsi qu'en étudiant des arbres récemment abattus au Nouveau-Mexique, en Californie, au Canada et même au nord de l'Europe, on a constaté sans peine le synchronisme de la largeur ou de la corrélation plus ou moins accusée) des anneaux avec la fréquence des taches solaires au cours des années correspondantes (graphique YII).

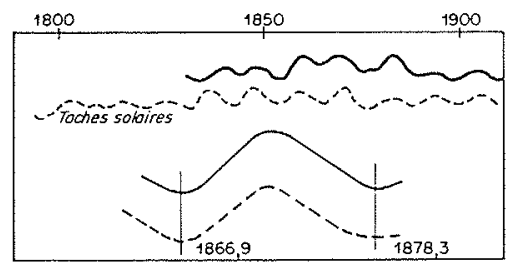

Graphique VII

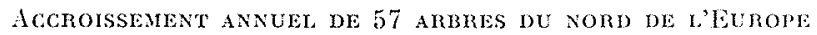
ET XOMBRES CORRESPONDANTS DES TACHES SOrames. Moyennes d'une période undecennale

«L'abondance des pluies, facteur principal de l'intensité de la végétation, est ici l'élément prépondérant : aussi sont-ce les régions ou il pleat rarement et où le sol est naturellement aride, ce qui accroît l'influence relative des fluctuations d'origine extérieure - qui se prêtent le mieux à une semblable étude (cette remarque pourrait être invoquée contre l'influence des taches solaires sur les précipitations).

«... Un point noir subsistait pourtant. On avait remarqué, de 1670 à 1720 , une lacune assez inquiétante : entre ces deux dates, les anneaux se distinguaient à peine les uns des autres et semblaient tous pareils. Or les annales astronomiques de la fin du xvr siecle nous apprennent précisément que, de 1645 a 1715 , le soleil n'a presque pas présenté de taches : la lacune constatée, loin d'infirmer la méthode, la corroborait brillamment.

«Elle a èté également confirmée par l'étude d'arbres plus anciens : poutres de maisons provenant des ruines de villages abandonnés depuis des siècles : une douzaine d'arbres étudiés par M. Dovgras remontent à 200 ans avant J.-C., 4 à 1.100 ans et 1 à 1.300 ans : sur tous le cycle des taches solaires élait visible.

«Il y a plus : certains glaciers du nord du Canada, soit dans les solitudes limitrophes de l'Alaska, soit dans le delta du Mackenzie, sont en lente rétrogradation. Ils laissent donc à nu des terrains recouverts par eux pendant des millénaires. Or, on a trouvé dans le sol sous-jacent, en divers endroits, des trones d'arbres que M. Ralph E., de Lury, a examinés et qui ont révélé eux aussi, par l'aspect de leurs anneaux concentriques, l'existence d'une période de 11 ans et quelques mois. A quelle époque ont récu ces arbres? Des géologues, après examen des lieux, ont parté de 300.000 ans : il n'est de toute facon pas douteux, si l'on se fonde sur les vitesses de rétrogradation actuellement observées des glaciers en cause, qưil s'agit là de plusieurs centaines de milliers d'années.

«Ainsi donc les taches du soleil existaient dejà, avec leur rythme actuel, en ces âges anciens. »

\section{Et M. Boster de conclure :}

"Nous sommes amenés par là à prèter davantage d'attention à des notions qu'il y a seulement vingt ans paraissaient assez aventurées. On a effectué, par exemple, des slatistiques en vue d'établir que, dans certains pays, les moissons, les crues périodiques de certains grands fleuves, les épidémies du bétail ou mème de la race humaine ou bien d'autres phénomènes étaient en relation avec les taches solaires. Tout cela n'est pas devenu nécessairement vrai; mais ce que nous venons de dire prouve tout au moins que ces problemes méritent d'être examinés d'un peu près, en dehors de tonte idée préconçue : c'est à la patience de nos successeurs qu'il appartiendra sans doute de les élucider définitivement. »

\section{Fluctuations périodiques dans le règne animal.}

D’un article récent «Au pays des fourrures La vie sauvage au Canada » paru dans la Tribune de Geneve du 5-6 septembre 1945, sous la signature d'Egmond d'Arcrs, nous lisons les lignes suivantes :

«Les récentes études sur le nombre des lynx et autres animaux au Canada, entreprises par le «Bureau of Animal Population » d'Oxford, en collaboration avec divers départements administratifs du gouvernement canadien, études qu'on a pu faire remonter à 1736 sans interruption les archives de la Compagnie de la Baie d'Hudson mentionnant en effet toutes les ventes de fourrures faites depuis cette date - ont conduit à ces constatations que d'importantes fluctuations se produisaient dans la quantité de lynx vivant dans certaines régions, que ces fluctuations interviemnent avec une régularité remarquable, que c'est en moyenne tous les 9 ans $1 / 2$ que les lynx sont les plus nombreux, que bien que les cycles soient partout d'une mème durée, les périodes durant lesquelles ces phénomenes se produisent varient grandement dans les différentes parties du pays et que le nombre des lynx peut atteindre son maximum à l'est, tandis qu'il est à son niveau le plus bas dans les régions occidentales.

"Bien qu'aucune étude approfondie de l'alimentation du lynx n'ait été entreprise jusqu'ici, on a tout lieu de croire que c'est le lapin à raquetles qui constitue la base de sa notrriture, et 
tout porte a croire (?) que le nombre de ces animaux varie également par cycle de 9 ans $1 / 2$. Les observations faites au cours des douze dernieres années (?) prouvent en effet que les variations coincident de très près chez les deux espèces.

«Des renseignements concernant les changements survenus ces dernières années dans le nombre des animaux (lapins de Norvège, renard arctique, hibou des neiges) ont été recueillis au moyen de questionnaires (?) envoyés chaque année aux trappeurs et aux postes de police montée, disséminés dans les régions septentrionales du Canada... Il semble dès à présent probable que chez ces animaux des cycles se manifestent également, de quatre années cette fois. La chose est parfaitement établie en ce qui concerne le renard.

"Aucun indice ne permet actuellement d'établir quel est le facteur qui détermine ce prodigieux rythme de la vie sauvage des habitants des forêts septentrionales : il est probable toutefois que les fluctuations climatiques jouent à cet égard un rôle important... »

O statistique, que de suppositions on fait en ton nom! C'est dommage qu'on n'ait pas trouvé une période de onze ans. En tout cas, voilà des horizons nouveaux ouverts aux chercheurs de périodes.

\section{VI}

L'avenir - qui, selon le poète, n'est à personne, mais à Dieu - nous dira peut-être un jour s’il existe d'autres périodicités que colles du Jour, de l'Année et des taches solaires.

En attendant, nous ne pouvons qu'avouer que la statistique mathématique ne nous permet de déceler que des fluctuations plus ou moins variables dans leurs caractéristiques (la périodicité de taches solaires n'échappe pas à cette restriction) sur lesquelles il n'est pas possible d'étayer des prévisions d'une grande utilité.

« La Physique - a écrit L. de Brograe - ne peut pas prédire les événements futurs avec exactitude, mais seulement les événements possibles et leurs probabilités relatives. »

La prévision des faits futurs n'est que le résultat d'un calcul de probabilité, permettant de prévoir, par exemple, avec une erreur $E$ la valeur prochaine d'un facteur $\mathrm{Y}$ :

$$
\mathrm{Y}=\mathrm{M} \pm \mathrm{E}
$$

Tout ce que nous pourons espérer et tenter de faire, c'est de substituer, à la valeur moyenne statistique $M$ - compte tenu de certaines corrélations, soit physique, $Y^{\prime}=f(X, Z)$, soit périodique, $\mathrm{Y}^{\prime \prime}=f(t)$, une valeur moyenne plus probable, variable en fonction des facteurs $X, Z$... ou du temps.

$$
\begin{array}{ll}
\mathrm{Y}^{\prime}=f(\mathrm{X}, \mathrm{Z} \ldots) \pm \varepsilon^{\prime} & \text { (corrélation physique) } \\
\mathrm{Y}^{\prime \prime}=l(t) \pm \varepsilon^{\prime \prime} & \text { (corrélation périodique) }
\end{array}
$$

La prévision sera d'autant plus efficace que les $\varepsilon^{\prime} \ldots \Sigma^{\prime \prime}$ seront plus petits par rapport aux $\mathrm{E}$.

Ainsi en est-il - tout au moins pour certaines régions, et certains facteurs - de la périodicité annuelle qui permet de réduire l'amplitude de la courbe des écarts, en substituant à la valeur. moyenne générale $\mathrm{M}$ des valeurs, soit mensuelles, soit même journalières, particulières, plus localisées dans le temps.

Mais en dehors de cette périodicité (et celle du jour), aucune autre périodicité n'a été trouvée qui permette d'améliorer d'une manière sensible les prévisions basées purement et simplement sur la distribution statistique de la variable $\mathrm{Y}$ considérée, prévisions se limitant à la connaissance des valeurs $Y$ possibles et de leurs probabilités respectives.

Nous avons traité par ailleurs la question des corrélations physiques et des prévisions qu'on peut baser sur certaines d'entre elles, en hydrologie, prévisions se limitant d'ailleurs à de courtes durées.

Mais qu'il s'agisse de corrélations périodiques ou physiques, n'oublions jamais que s'il y a, certes, de telles corrélations dans la nature qui nous sont accessibles, il y a aussi des coïncidences fortuites, dues à des relations dont nous ne pouvons connaître ni les éléments ni même la nature.

Et si les années, du point de vue météorologique, hydrologique, se succedent suivant un ordre supérieurement établi, nous devons avouer que cet ordre, certes déterminé, est pour nous indéterminable, n'étant pas déterminé nécessairement « mécaniquement»: « La nature ne nous présente pas de lois causales, mais de simples lois de probabilité : le déterminisme absolu, à supposer qu'il existe, est absolument inaccessible. »

Un savant a pu partir du Libre arbitre des Atomes qui, a même ajouté un autre savant, n'exclut pas le miracle.

Pour nous, les années ne se succèdent-elles pas comme si elles jouissaient d'un certain « libre arbitre $»$ ?

"Nos lois n'expriment que le comportement moyen d'objets dont nous ne pouvons définir le comportement individuel. Notre science et notre expérience qui se fondent sur elles sont impuissants à franchir le seuil du domaine où la $\mathrm{Na}$ ture agit en nous dérobant le principe de ses œuvres et le secret de sa spontanéité créatrice. ”

Et c'est très bien ainsi, car s'il en était autrement, le Monde ne serait plus le Monde, l'Homme ne serait plus l'Homme. 